\title{
The role of Sulforaphane in cancer chemoprevention and health benefits: a mini-review
}

\author{
Reza Bayat Mokhtari ${ }^{1,2,3,4}$ - Narges Baluch ${ }^{5}$ - Tina S. Homayouni ${ }^{1}$. \\ Evgeniya Morgatskaya $^{1} \cdot$ Sushil Kumar $^{1} \cdot$ Parandis Kazemi $^{1} \cdot$ Herman Yeger $^{1,2,3,4}$
}

Received: 4 July 2017 / Accepted: 6 July 2017 /Published online: 23 July 2017

(C) The International CCN Society 2017

\begin{abstract}
Cancer is a multi-stage process resulting from aberrant signaling pathways driving uncontrolled proliferation of transformed cells. The development and progression of cancer from a premalignant lesion towards a metastatic tumor requires accumulation of mutations in many regulatory genes of the cell. Different chemopreventative approaches have been sought to interfere with initiation and control malignant progression. Here we present research on dietary compounds with evidence of cancer prevention activity that highlights the potential beneficial effect of a diet rich in cruciferous vegetables. The Brassica family of cruciferous vegetables such as broccoli is a rich source of glucosinolates, which are metabolized to isothiocyanate compounds. Amongst a number of related variants of isothiocyanates, sulforaphane (SFN) has surfaced as a particularly potent chemopreventive agent based on its ability to target multiple mechanisms within the cell to control carcinogenesis. Anti-inflammatory, pro-apoptotic and modulation of histones are some of the more important and known
\end{abstract}

The original version of this article was revised: The Forsyth Institute was removed from the institution list of Reza Bayat Mokhtari. The given name of the 4th author was corrected from Zhenya to Evgeniya.

\footnotetext{
Reza Bayat Mokhtari

reza.mokhtari@sickkids.ca

Herman Yeger

hermie@sickkids.ca

Narges Baluch

15nb15@queensu.ca

Tina S. Homayouni

tina_homayouni@hotmail.com

Evgeniya Morgatskaya

zhenya.morgatskaya@gmail.com

Sushil Kumar

sushil.kumar@sickkids.ca
}

mechanisms by which SFN exerts chemoprevention. The effect of SFN on cancer stem cells is another area of interest that has been explored in recent years and may contribute to its chemopreventive properties. In this paper, we briefly review structure, pharmacology and preclinical studies highlighting chemopreventive effects of SFN.

Keywords Chemopreventive agents · Isothiocyanates · Sulforaphane

\section{Introduction}

Chemopreventive agents tend to limit the incidence and growth of cancer by reversing or suppressing carcinogenesis (Sheth et al. 2015). "Green chemoprevention" is defined as consumption of whole plant foods or their pure extracts to prevent cancer (Fahey et al. 2012). An emerging importance of research on plant foods

Parandis Kazemi

thrhbd@yahoo.com

1 Developmental and Stem Cell Biology, The Hospital for Sick Children, Toronto, ON, Canada

2 Department of Paediatric Laboratory Medicine, The Hospital for Sick Children, Toronto, ON, Canada

3 Institute of Medical Science, University of Toronto, Toronto, ON, Canada

4 Sickkids Research Center, Peter Gilgan Centre, 686 Bay St., Rm 15.9714, Toronto, ON M5G 0A4, Canada

5 Department of Pathology and Molecular Medicine, Richardson Laboratory, Queen's University, 88 Stuart Street, Kingston, ON K7L 3N6, Canada 
and cancer prevention suggests the potential beneficial effect of a diet rich in cruciferous vegetables (AICR 2007). The Brassica family of cruciferous vegetables, including broccoli, is a rich source of glucosinolates. Isothiocyanates are the hydrolytic product of the glucosinolates. Amongst a number of closely related variants of isothiocyanates, sulforaphane (SFN) is the most potent chemopreventive agent that has been shown to target multiple mechanisms within the cell (Zhang et al. 1992). Glucoraphanin [4-methylsulfinylbutyl glucosinolate] is the glucosinolate prevalent in broccoli, which is the precursor of SFN (Cramer and Jeffery 2011).

\section{Chemical structure and properties}

SFN is a molecule within the isothiocyanate group of organosulfur compounds. Isothiocyanates are the hydrolytic products of anionic glucosinolate compounds produced by the endogenous thioglucosidase enzyme also known as myrosinase. Glutathione and myrosinase are physically separated in intact plant cells (Dinkova-Kostova et al. 2006). Upon physical damage to the plant, these two compounds come in contact with each other and myrosinase removes the $\beta$-thioglucose moiety from glucosinolate, leading to the formation of a variety of unstable compounds. Depending on the R group and the reaction condition, a mixture of final products are formed, including epithionitriles, nitriles, isothiocyanates, thiocyanates, and oxazolidine-2-thiones (Fig. 1). In broccoli, the primary glucosinolate is glucoraphanin (4-(methylsulfonyl) butyl glucosinolate) which yields SFN, isothiocyanate-(4R)-(methylsulfonyl) butane and SFN nitril, (4-(methylsulfonyl) butyl (Matusheski and Jeffery 2001). When purified glucosinolates and myrosinase are incubated together at neutral $\mathrm{pH}$, isothiocyanates are the only products of this reaction (Srivastava and Hill 1974). Nevertheless, only SFN, and not SFN nitrile, was shown to possess chemoprotective properties through induction of phase II detoxification enzymes. Musheski et al. administered SFN and SFN nitrile to male Fischer rats with daily doses of 200,500 or $1000 \mu \mathrm{mol} / \mathrm{kg}$ for five days. They showed that high dose SFN, but not SFN nitrile, induced hepatic, colonic, mucosal and pancreatic quinone reductase and GST activities (Matusheski and Jeffery 2001).

SFN molecule consists of an isothiocyanate functional group $(-\mathrm{N}=\mathrm{C}=\mathrm{S})$ and a methylsulfonyl side chain (R-(SO)-R) (Fig. 1). Zhang et al. synthesized different racemic analogs of SFN differing in the oxidation state of sulfinyl group and the number of methylene groups. The potencies of each molecule in the induction of phase II detoxification enzymes were measured. Compared to the sulfide and sulfone analogs, SFN had the greatest potency at inducing phase II enzymes (Zhang et al. 1992). Also, due to its electrophilic structure and lack of aromatic groups, SFN is water-soluble, and its pharmacological activity is better at the neutral $\mathrm{pH}$ of the intestine (Xu et al. 2012).

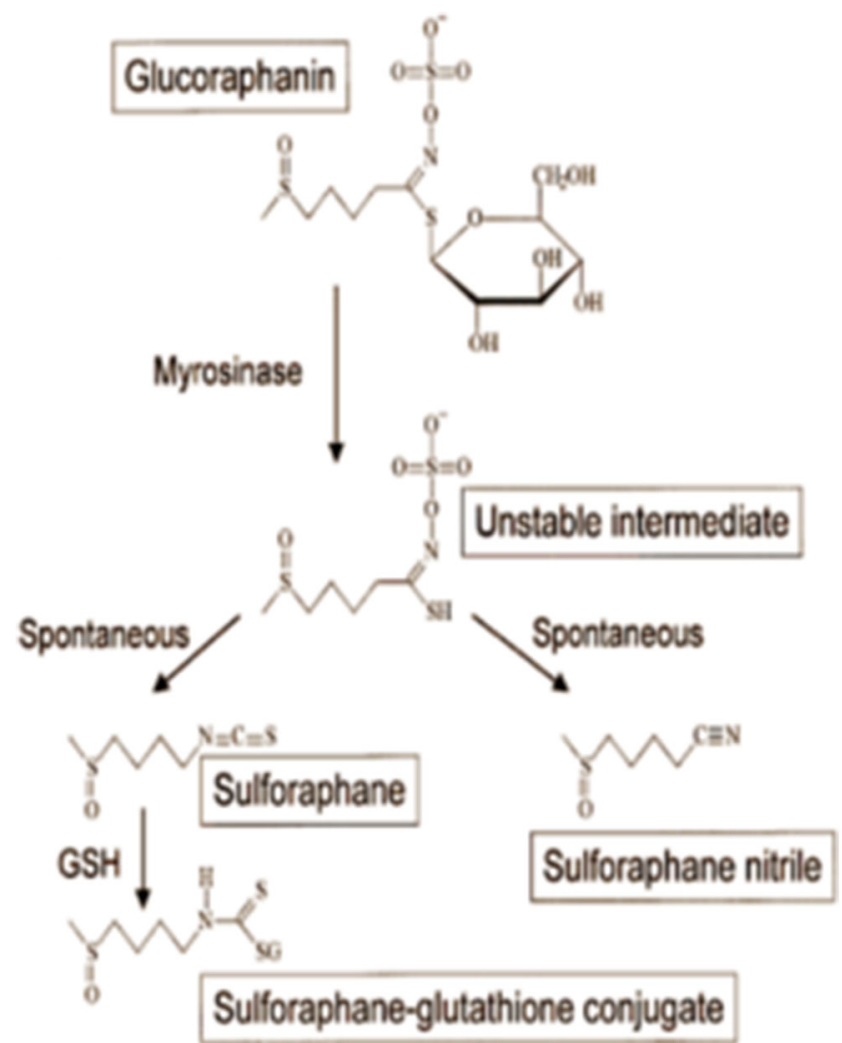

Fig. 1 The production of the detoxifying enzyme glutathione by activation of Sulforaphane: The figure demonstrates that the physical damage to the plant and the activation of myrosinase lead to the formation of some unstable compounds. The $\mathrm{R}$ group and the reaction condition determine the final products

\section{Pharmacology}

\section{Metabolism}

In addition to the myrosinase enzyme, which hydrolyzes the glucoraphanin to SFN, glucoraphanin is also degraded by gut microbiota and absorbed in the colon (Luang-In et al. 2014). In current studies, particular emphasis is placed on the glutathionedependent pathway for the metabolism of SFN. First, because there is a high millimolar concentration of glutathione (GSH) in tissues, the conjugation with SFN is a common occurrence (Dinkova-Kostova et al. 2006). GSH conjugation is a major route for elimination of isothiocyanates in mammals (Kassahun et al. 1997). GSH conjugation is catalyzed by glutathione transferase (GSTs), which lowers the pKa value of the cysteine residues of GSH such that it exists as a thiolate anion in physiological condition; this enhances its nucleophilic properties to attach to other electrophiles, including the central carbon of SFN (DinkovaKostova et al. 2006). After conjugation, SFN-GSH is enzymatically degraded to SFN-cysteinglycine. Further degradation yields SFN-cysteine that is eventually acetylated to form SFNN-acetylcysteine or SFN-NAC. SFN-NAC is a bioactive compound that reacts with thiol groups of amino acids residues of 
different proteins (Kumar and Sabbioni 2010). The metabolites are then excreted in urine and bile (Kassahun et al. 1997).

\section{Bioavailability and biotransformation}

There are several factors that affect the bioavailability of SFN. When myrosinase acts on glucoraphanin to produce primary unstable products, epithiospecifier protein (EPS) present in the plant directs the hydrolysis of glucoraphanin towards SFN nitrile formation, which does not have the anticarcinogenic properties of SFN. Matusheski et al. demonstrated that mild heating simultaneously decreased ESP activity resulting in, an increased level of SFN relative to SFN nitrile. He also argued that genetic manipulation, to eliminate the expression of ESP, can also increase the conversion of glucoraphanin to SFN (Matusheski and Jeffery 2001).

Another factor that affects the bioavailability of SFN is the reversible endogenous biotransformation of SFN metabolites to structurally related erucin metabolite. Erucin is also metabolized through the same mercapturic acid pathway (Kassahun et al. 1997). Kassahun and collogues investigated the metabolic fate of SFN in rats. They administered single doses of $50 \mathrm{mg} / \mathrm{kg}$ of SFN to rats and analyzed their bile and urine. The samples were screened for GSH and NAC conjugates. In bile five thiol conjugates were detected, including GSH conjugates of SFN (delta SFN) and erucin which suggested oxidative metabolism of SFN, and NAC conjugate of SFN and erucin (Kassahun et al. 1997). These findings suggest that it is crucial to include all the biotransformed metabolites of SFN such as erucin for comprehensive analysis of health benefits and pharmacology of SFN.

\section{Pharmacodynamics and mechanisms of action}

SFN targets various pathways within the cell to attenuate, reverse, or block the effect of carcinogens. SFN exerts primary chemoprevention by inhibiting phase 1 metabolizing enzymes (mostly cytochrome P450) (Langouet et al. 2000), inducing activation of phase 2 detoxification enzymes and suppressing pro-inflammatory responses within the cell. SFN promotes secondary chemoprevention by modulating epigenetic changes within the cell that promote transcription of apoptotic and cell cycle arrest genes, such as p21 and cyclin D1. Finally, SFN exerts tertiary prevention through abrogating tumorigenesis and metastatic progression by targeting cancer stem cells in pancreatic and prostate cancer (Kallifatidis et al. 2011).

\section{Induction of Nrf2-ARE mediated transcription}

Keap1-Nrf2 is an intrinsic signaling pathway that provides cytoprotective response towards the disruption of oxidative status of the cell, normally elicited by intrinsic and extrinsic stress (Kwak et al. 2004). The current model for the Keap1-Nrf2 interaction state under homeostatic conditions indicates that $\mathrm{Nrf} 2$ is bound by Keap1, which allows for the ubiquitination of Nrf2, marking it for proteasomal degradation. Interaction of SFN with one of the protein co-factors of Keap1, Cul3, disrupts ubiquitination of $\mathrm{Nrf} 2$, thus releasing $\mathrm{Nrf} 2$ to translocate to the nucleus and bind the antioxidant response elements of many functional phase 2 and anti-oxidant genes (Fig. 2a) (Kwak et al. 2004). These genes include $\mathrm{NAD}(\mathrm{P}) \mathrm{H}$ quinone reductase 1 (NQO1), glutathione S-transferases (GSTs), heme oxygenase 1, thioredoxin reductase, and also-keto reductases (Fig. 2a) (Kwak et al. 2004). In an in vivo study, a broccoli sprout-derived beverage of $600 \mu \mathrm{mol}$ glucoraphanin and $40 \mu \mathrm{mol}$ SFN were provided to 191 individuals highly exposed to air pollutants. Urinary excretion of the mercapturic acid derivatives of pollutants such as benzene acrolein and crotonaldehyde were measured before and during SFN intake. The results showed a significant increase in the level of excretion of glutathione-derived conjugates of all airborne metabolites, confirming that SFN enhances detoxification of some airborne carcinogens (Egner et al. 2014).

\section{Suppression of the inflammatory response}

Initiation of the inflammatory response within cells requires initiation of several signaling pathways that promote expression of various cytokines in response to endotoxins. Two important pathways related to the inflammatory response include nuclear factor kappa B (NFkB) and mitogen-activated protein kinases (MAPK) signaling pathways (Shibata et al. 2010, Ben-Neriah and Karin 2011). NFkB signaling pathway in particular, is mechanistically part of the innate immunity response and is conserved in all multicellular organisms, which further demonstrates its importance in inflammatory reactions (BenNeriah and Karin 2011). NFkB signaling pathway has a twofold role in cancer initiation and progression. Firstly, NFkB activity prevents tumor initiation by removing cells that have transformed through mutation or carcinogens through its interactions with cytotoxic immune cells (Disis 2010). Ligand binding to TLR4 leads to the activation of two parallel pathways, the MyD88 dependent and independent pathways that together, lead to the activation of NF-kB (Fig. 2b).

Moreover, the second function of NFkB in cancer progression is that once tumor formation has already initiated, NFkB becomes highly active and prolongs its progression by upregulating anti-apoptotic genes, or indirectly upregulating cellular proliferation (Jost and Ruland 2007). As a result, NFkB signaling pathway plays an important role in cancer progression and subsequently has become a therapeutic target. Thus, suppressing inflammation would theoretically inhibit carcinogenesis and definitively tumor development.

SFN is depicted to have an anti-inflammatory role in conjunction with its other cancer-preventive properties. SFN has been shown to form adducts with cysteine residues in the extracellular domain of TLR4 thus inhibiting activation and promotion of the downstream NFkB mediated signaling pathway 


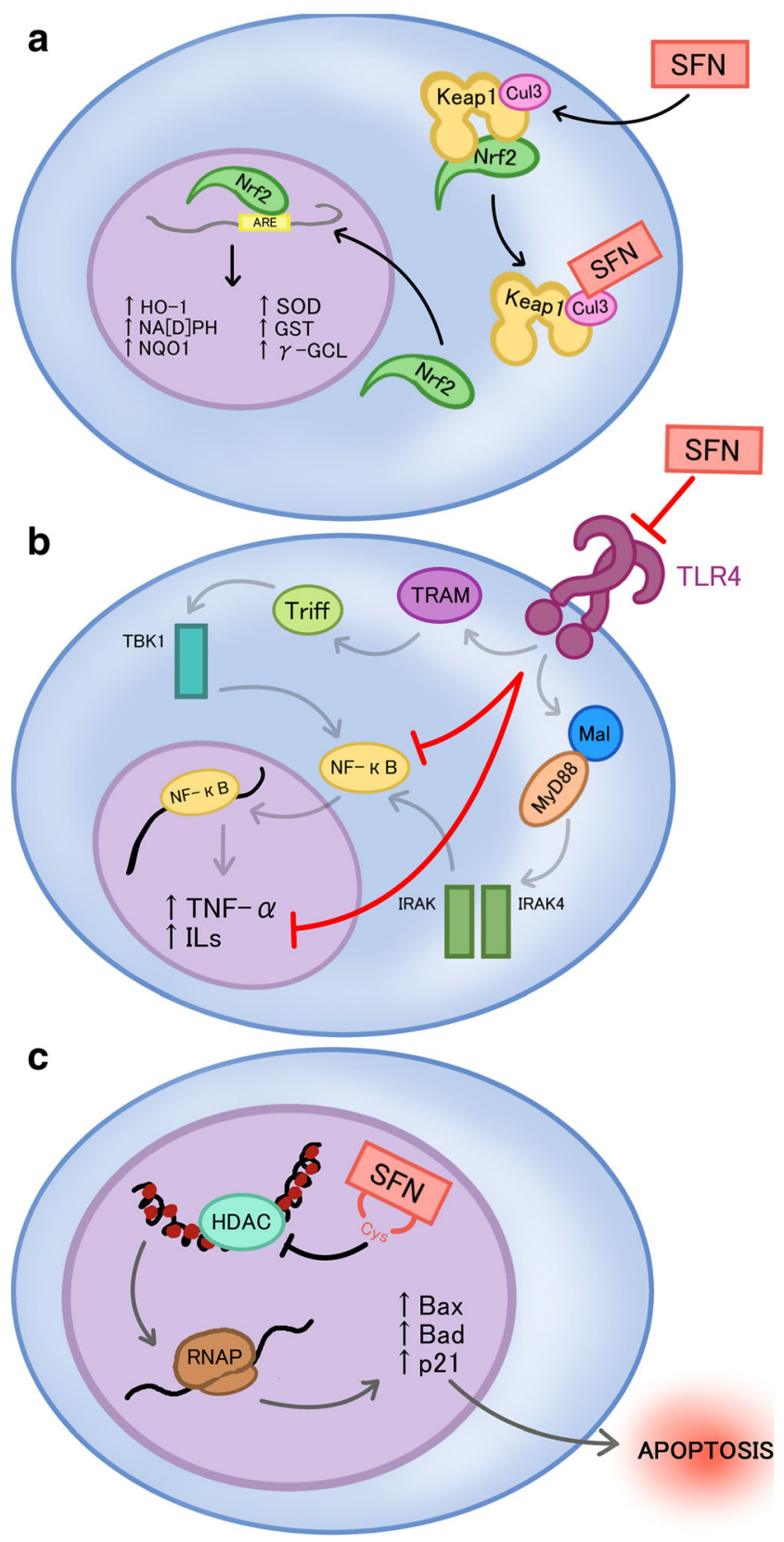

Fig. 2 The anti-inflammatory, chemoprotectant, and epigenetic modulatory activities of SFN: a Sulforaphane (SFN) binds to the Cul3 cofactor of kealch-like ECH-associated protein 1 (Keap1), leading to the release of nuclear factor erythroid 2-related factor 2 (Nrf2) and its translocation to the nucleus to elicit transcription of various phase 2 detoxifying genes, which include but are not limited to $\mathrm{NAD}(\mathrm{P}) \mathrm{H}$ quinone reductase 1 (NQO1), glutathione S-transferases (GSTs), and heme oxygenase 1 (HO-1). b SFN prevents inflammatory activation by preventing the transcription of inflammatory related genes, such as tumor necrosis factor-alpha (TNF-alpha) and interleukins (ILs), through the inhibition of toll like receptor 4 (TLR4) and its subsequent signaling cascade. c SFN inhibits histone deacetylase, which eventually leads to the increase of transcription of pro-apoptotic factors, such as Bax, Bad, and $\mathrm{p} 21$, and subsequently apoptosis

(Fig. 2b) (Youn et al. 2010). Activation of NFkB in prostate and colon cancer is associated with up-regulation of inflammatory cytokines and anti-apoptotic genes. It was also demonstrated that NFkB was constitutively active in patients with lymphoma and solid cancers such as prostate, breast, oral cavity, liver, colon, pancreas and ovary (Aggarwal and Gehlot 2009). SFN has been shown to down-regulate NFkB in $\mathrm{PC} 3$ prostate cancer cells (Choi et al. 2007). Heiss et al. demonstrated that SFN reduced DNA binding of NFkB without interfering with lipopolysaccharide induced degradation of the inhibitor of NFkB and nuclear translocation of NFkB (Heiss et al. 2001).

\section{Epigenetic modification of histones}

Epigenetic changes play a critical role in development and differentiation phases; however, they can also arise in mature humans (Jaenisch and Bird 2003). Compared to genetic alterations, epigenetic changes can be modulated (Nian et al. 2009). Histone modifications and DNA methylation are the main molecular mechanisms that constitute the epigenetic process as modulated by HDAC and methyltransferases (DNMT), respectively (Gal-Yam et al. 2008). Histone acetylation results in an open chromatin structure that facilitates the access of transcription factors to specific gene loci while HDAC performs an opposite action by removing the acetyl group from the histones. Overexpression and/or over activity of HDAC are common hallmarks of cancer (Fraga et al. 2005). This can lead to a loss of transcription of regulatory genes responsible for apoptosis and cell cycle arrest in cancer cells.

SFN can act as an HDAC inhibitor (Nian et al. 2009), which may lead to its ability to induce cell cycle arrest and apoptosis (Fig. 2c). Computer modeling predicts that SFNCys is a good fit for the active site of the HDAC enzyme (Nian et al. 2009). Zuryn et al. used image-based fluorescence cytometry to show that in cells treated with SFN, cyclin D1 content was increased at $30 \mu \mathrm{mol}$ SFN but was decreased at $60 \mu \mathrm{M}$ and $90 \mu \mathrm{M}$ SFN (Zuryn et al. 2016). The highest increase in the number of $\mathrm{p} 21$ positive cells (apoptotic cells) occurred at $60 \mu \mathrm{M}$ SFN. Furthermore, they showed that transfection of cells with cyclin D1-siRNA resulted in significant change in the fraction of apoptotic cell population, highlighting the importance of cyclin D1 in the therapeutic effect of SFN (Zuryn et al. 2016).

In addition to epigenetic modification of histones, SFN has been shown to modulate non-coding RNAs. MicroRNAs (miRNAs), a subtype of non-coding RNAs, can both modulate and function as tumor suppressor or oncogenes (Tortorella et al. 2015). Treatment of breast ductal carcinoma in situ stemlike subpopulations with SFN increased exosomal miR-140 and decreased miR-21 and miR-29. In addition, SFN decreased colony formation and ALDH1 expression. Increased miR-140 is associated with changes in the microenvironment leading to cell signaling alterations (Tortorella et al. 2015). Furthermore, miR-140 has been suggested to target stem cell factors, SOX9 and ALDH1 (Tortorella et al. 2015). 


\section{Apoptotic signaling pathway}

Carcinogens can create distinct molecular changes in cellular homeostasis that lead to uncontrollable cellular division, highlighting the anti-apoptotic activity of cancer (Rudolf et al. 2014). Targeting pro-apoptotic factors is one strategy to induce apoptosis and therefore prevent tumor formation from commencing. In one study, SFN was shown to induce apoptosis involving p53 and p38, and to downregulate antiapoptotic factors Bcl-2, caspase 8 and Bid that are part of the intrinsic signaling pathway in melanoma cells (Rudolf et al. 2014). SFN's chemopreventative properties was also demonstrated in another study, where through its HDACi activity, SFN subsequently induced apoptosis in PC-3 human prostate cancer cells. Specifically, SFN activated caspases 3, 8 and 9 and downregulated anti-apoptotic factor Bcl-2 (Singh et al. 2004). Additionally, SFN was also shown to induce apoptosis in U251MG glioblastoma cells, and accordingly downregulated Bcl-2 and upregulated the expression of Bax, Bad, and Bax/Bcl-2 ratio (Zhang et al. 2016a, b). As well, SFN was also demonstrated to target and activate the MEK/ERK signaling pathway, an important pathway involved in cell survival, proliferation, differentiation and apoptosis in malignant glioma GB8401 cell line. As a result, activation of SFN led to $\mathrm{MEK} / \mathrm{ERK}$-mediated apoptosis (Huang et al. 2012).

\section{Sulforaphane and angiogenesis}

Angiogenesis refers to the formation of new blood vessels from pre-existing microvasculature and has a crucial role in tumor malignant transformation and progression (Benazzi et al. 2014). Inhibitory effects of SFN on angiogenesis have been shown on HMEC-1, an immortalized human microvascular endothelial cell line. In vitro treatment of HMEC-1 cells with SFN significantly decreased the formation of new microcapillaries (Bertl et al. 2006). SFN inhibits hypoxiainducible factor-1 $\alpha(\mathrm{HIF}-1 \alpha)$ and c-Myc, two angiogenesisassociated transcription factors. The inhibition of these transcription factors results in the inhibition of key angiogenesis factors, vascular endothelial growth factor (VEGF) and matrix metalloproteinase-9 (MMP-9); thus, endothelial cell basal membrane integrity is affected by SFN and subsequently, angiogeneic and metastatic potential is reduced (Fig. 3a) (Bertl et al. 2006). This mechanism is further supported in another study in HCT116 human colon cancer cells, where SFN significantly reduced the expression level of VEGF and HIF- $1 \alpha$ in addition to the migratory potential of HCT 116 cells (Kim et al. 2015).

\section{Sulforaphane and autophagy}

Autophagy is a catabolic process that degrades damaged proteins and organelles, thereby maintaining the quality of cells.

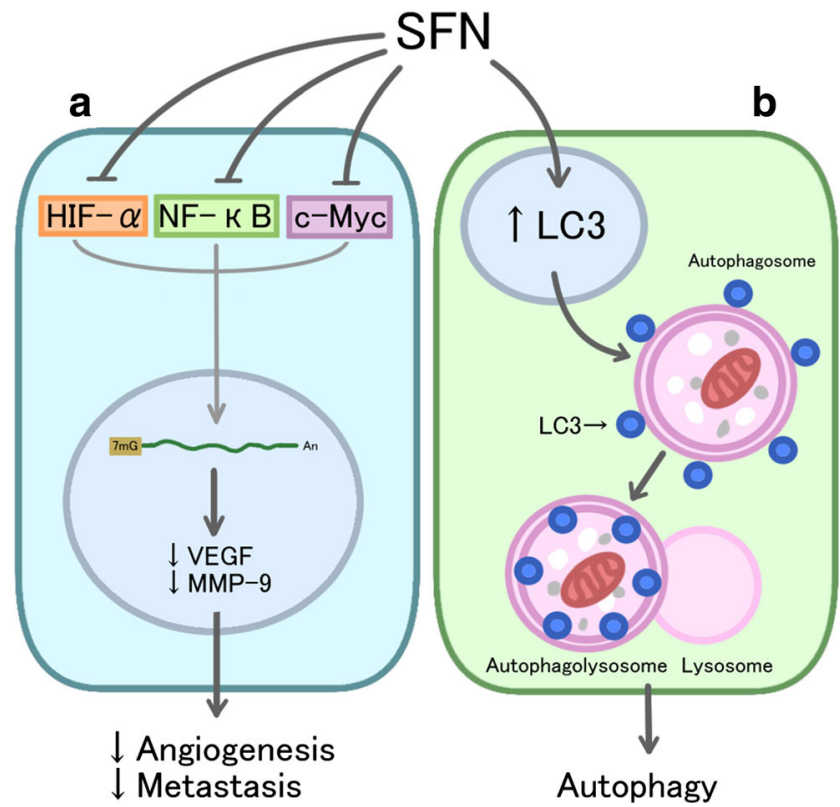

Fig. 3 The anti-angiogenesis, anti-metastasis and autophagy activities of SFN: a Sulforaphane (SFN) inhibits transcription factors hypoxiainducible factor (HIF)-1alpha, nuclear factor-kappaB (NFkB), and proto-oncogene myc (c-Myc), resulting in the downregulation of key angiogenic and metastatic regulators, vascular endothelial growth factor (VEGF) and matrix metallopeptidase 9 (MMP-9), and thus, the reduction of angiogenic and metastatic potential. b SFN induces the recruitment and increases the expression of LC3 to autophagosomes, thus, increasing the activation of the autophagy pathway

It has been suggested that autophagy, through a cytoprotective mechanism, can help cancer cells to survive. SFN has shown to induce autophagy by increasing expression of LC3 as well as upregulating its recruitment to the autophagasome (Fig. 3b) (Antosiewicz et al. 2006). The effect of SFN on autophagy has been studied in a few types of cancer. In PC-3 and LNCaP human prostate cancer cell lines, treatment with SFN induced autophagy, which appears to be a defense mechanism against SFN-induced apoptosis. However, further treatment of cells with 3-methyladenine, an inhibitor of autophagy, enhanced cytosolic release of cytochrome $c$ and apoptotic effects of SFN (Antosiewicz et al. 2006). It has been further shown that the combination of SFN with bafilomycin A1, as an inhibitor of autophagy, enhances apoptotic effect of SFN in breast cancer cell line MDA-MB-231 (Kanematsu et al. 2010).

\section{Sulforaphane and metastasis}

Tumor invasion and metastasis is a multi-stage process that is associated with poor prognosis and high morbidity and mortality. One crucial step of tumor invasion and migration involves degradation of extracellular matrix (ECM). Matrix metalloproteinases (MMPs) are an important group of matrix degrading enzymes responsible for tumor cell migration and invasion. It has been observed that MMP-2 and MMP-9 are particularly important in the process of tumor invasion, since 
they can degrade type IV collagen and ECM (Tsuchiya et al. 1994). Isothiocyanates including SFN have shown inhibitory effect on MMP-9 expression and activity in C6 glioma cells. In the same study, the wound-healing assay and Matrigelbased Transwell invasion assay confirmed the inhibitory effect of SFN on C6 glioma cell invasion and migration at a concentration of $10 \mu \mathrm{m}$ (Lee et al. 2015). In another study SFN inhibited lung metastasis of the highly metastatic B16F-10 melanoma cells in C57BL/6mice by $95.5 \%$. SFN also improved the survival of animals with metastasis by $94.06 \%$. These effects were attributed to inhibition of activation of MMPs (Fig. 3a) (Thejass et al. 2006). Similarly, SFN inhibited the invasion and metastasis of oral carcinoma cell line YD10B. Dose-dependent decrease in expression of MMP-1 and MMP-2 was observed (Jee et al. 2011).

\section{Therapeutic application and health benefits}

Over the last few decades, an emerging emphasis on the importance of dietary phytochemicals to fight cancer has been spurred on by the limited efficacy and various side effects associated with chemotherapy and radiotherapy (Singh et al. 2017). In addition, health benefits of dietary phytochemicals including cruciferous vegetables has been observed in epidemiological studies. It has been suggested that, compared to other vegetables and fruits, cruciferous vegetables may be more effective in cancer prevention. Most studies showed that the consumption of 3-5 servings of cruciferous vegetables per week has the strongest inverse relationship with cancer risk (Jeffery and Keck 2008). In The Nurses' Health Study 88,410 female registered nurses were followed for 14 years to investigate the association between fruit and vegetable intake and the incidence of non-Hodgkin's lymphoma. The results showed that women who had $>5$ servings of cruciferous vegetables per week had a lower risk of non-Hodgkin's lymphoma (multivariate Relative Risk: 0.67) (Zhang et al. 2009). In a case-control study involving 1619 cases of confirmed prostate cancer, intake of cruciferous vegetables was inversely related to prostate cancer, especially in advanced cases (Kolonel et al. 2000). In another case-control study it was shown that higher intake of cruciferous and dark green vegetables is associated with lower risk of renal cell carcinoma (RCC). Importantly, this study demonstrated a stronger association of RCC with cruciferous vegetables than RCC with nutrients such as alphacarotene, beta-carotene, beta-cryptoxanthin and lutein, which suggests presence of another effective compound in cruciferous vegetable (i.e. isothiocyanates) that is responsible for reduced risk of cancer (Yuan et al. 1998). Although epidemiological studies showed the health benefits of cruciferous vegetables, preclinical studies are required to reveal the underlying mechanisms. Isothiocyanates including SFN have been widely investigated in in vivo and in vitro studies. SFN has shown to selectively exert cytotoxic effects in various human cancer cells while having no cytotoxic effect on normal cells. Clarke et al. evaluated the effect of SFN on normal, benign hyperplasia and cancerous prostate epithelial cells. They observed that $15 \mu \mathrm{mol}$ SFN selectively inhibited HDAC activity and induced $\mathrm{p} 21$ expression in benign hyperplasia and cancerous cells, while it only caused a transient decrease in HDAC activity and did not affect $\mathrm{p} 21$ expression in normal cells, suggesting a differential effect on normal versus cancer cells (Clarke et al. 2011a, b). Experimental studies supported by epidemiological data suggest a chemoprotective association between consumption of SFN, contained in broccoli sprouts, and breast cancer. Estrogen is considered a risk factor in breast carcinogenesis, which operates through two mechanisms, first by the induction of estrogen-induced cell proliferation and second, by the formation of active estrogen metabolites. Oxidative metabolism of estrogens by CYP1B1 produces 4hydroxyestradiol, which generates free radicals that act as carcinogens (Licznerska et al. 2015). SFN induces NQO1 and GST that detoxifies these metabolites, suppressing carcinogenesis (Licznerska et al. 2015). SFN as a dietary supplement demonstrated cancer chemoprevention efficacy in intestinal polyposis in ApcMin/+ mouse model in a dose-dependent manner. Supplementation with SFN (300 and 600 p.p.m.) for 3 weeks significantly decreased the number and size of intestinal polyps. In addition, these polyps showed higher apoptotic and lower proliferative indices ( $\mathrm{Hu}$ et al. 2006). Topical application of SFN-containing broccoli sprout extract for 11 weeks reduced skin tumor burden, incidence and multiplicity by $50 \%$ in irradiated mice. However, this effect was only observed in the group of animals that received a maximum dose of $1 \mu \mathrm{M}$ SFN (Dinkova-Kostova et al. 2006).

Additionally, studies highlighting the ability of SFN to attenuate stemness properties of cancer stem cells are emerging. For instance, Vyas et al. demonstrated that SFN treatment diminished prostate cancer stemness traits, including the enhanced activity of aldehyde dehydrogenase 1 (ALDH1), enrichment of CD49f + fraction, and sphere forming efficiency. As well, the expression of c-Myc, an oncogenic transcription factor that is frequently deregulated in prostate cancer cells, was markedly suppressed in vivo and in vitro (Vyas et al. 2016). Moreover, evidence of SFN's anti-tumor effects is demonstrated in a study done by Li et al., using the breast cancer xenograft model of SUM159 cells in NOD/SCID mice. Xenografted mice that were injected with $50 \mathrm{mg} / \mathrm{kg}$ of SFN displayed a 50\% marked reduction in size compared to the control, while also displaying a reduced ALDH-positive population, an indicator of a decreased cancer stem cell (CSC) population (Li et al. 2010). In the same study, SFN was also shown to reduce the self-renewal ability of breast cancer stem cells, as indicated by the reduction in sphere-forming cells that are characteristically known to have stemness properties (Li et al. 2010). Furthermore, in a previous study, SFN was able to 
additively enhance the therapeutic effects of TRAIL (Tumour $\underline{\text { Necrosis }}$ Factor-Related Apoptosis-Induced Ligand) agent and consequently decrease the tumor initiating cells (de Conti et al. 2012) or the CSC population synergistically. Mechanistically, it was demonstrated that CSCs were resistant to the apoptotic inducing effects of TRAIL because of the presence of the enhanced signaling of NFkB (TRAILactivated nuclear factor-kappaB). Moreover, SFN is characteristically able to inhibit NFkB signaling and therefore reduce TRAIL treatment resistance and reduce viability of the CD44+/CD133+ CSC subpopulation (Tortorella et al. 2015).

Table 1 summarizes the preclinical studies of SFN. In addition to pre-clinical experiments, SFN has demonstrated promising results in clinical studies. Zhang et al. found an inverse association between cruciferous vegetable intake and Ki-67 protein expression in women with DCIS; however, similar results were not observed in benign breast tissue or invasive ductal carcinoma (Zhang et al. 2016a, b). A doubleblinded, randomized, placebo-controlled clinical trial that involved 78 patients with biochemical recurrence of prostate cancer after radical prostatectomy used a daily oral administration of $60 \mathrm{mg}$ of stabilized free SFN for 6 months followed by 2 months without treatment. Median log PSA slopes were calculated and showed a significant decrease in the treatment group. In addition, men that received treatment with SFN had a significant longer PSA doubling time compared with the placebo group (28.9 vs. 15.5 months). In men with recurrent prostate cancer treatment with $200 \mu$ moles/day of SFN-rich extracts for a maximum period of 20 weeks resulted in significant lengthening of the on-treatment PSA doubling time (6.1 months pre-treatment vs. 9.6 months on-treatment). Importantly, none of the study subjects experienced grade 3 adverse events. GI disorders were the most common adverse events observed in this study (Alumkal et al. 2015). An additional health benefit of SFN is its ability to display protective effects on the quality of life of cancer patients. Adjuvant chemotherapy has shown to decrease the quality of life in some cancer patients by inducing anxiety, depression and/or emotional dysfunction (Reece et al. 2013). Interestingly, there are studies demonstrating the anti-anxiety and anti-depressant effects of SFN. Wu et al. showed that SFN, due to its antiinflammatory effects, could inhibit inflammatory response to stress and inhibit hypothalamic-pituitary-adrenal stress axis (Wu et al. 2016). It is argued that due to SFN's low molecular weight, it can easily cross the blood brain barrier to produce anti-depressant and anti-anxiety effects in animal studies.

Significantly, a diet of three to five servings per week is sufficient to decrease the risk of cancer development by $30 \%$ $40 \%$ (Jeffery and Keck 2008). Systematic reviews of the literature have also confirmed an association with weekly intake of cruciferous vegetable and a decreased risk of cancer development (Wu et al. 2013). It was found that subjects who consumed at least one portion of cruciferous vegetables per week as compared with those with no or occasional consumption were associated with a significantly reduced risk of oral cavity, pharynx, esophageal, colorectal, breast, and kidney cancer.

Table 1 Pre-clinical studies of SFN in various human cancer cells

\begin{tabular}{|c|c|c|c|}
\hline Cell Lines & Dose of SFN & Key Observation & Reference \\
\hline $\begin{array}{l}\text { Benign hyperplasia (BPH1) and cancerous } \\
\text { prostate epithelial cells (LnCap and PC3) }\end{array}$ & $15 \mu \mathrm{M}$ & $\begin{array}{l}\text { - HDAC activity inhibition } \\
\text { - p21 induction }\end{array}$ & $\begin{array}{l}\text { (Clarke et al. 2011a, } \\
\text { b) }\end{array}$ \\
\hline $\begin{array}{l}\text { Breast cancer cell lines (MCF7 and } \\
\text { MDA-MB-231) } \\
\text { and non-tumorigenic MCF10A }\end{array}$ & $\begin{array}{l}5 \mu \mathrm{M} \text { and } \\
20 \mu \mathrm{mol} / \mathrm{L}\end{array}$ & $\begin{array}{l}\text { - Cell viability induction } \\
\text { - Apoptosis induction } \\
\text { - CYP1A1 reduction } \\
\text { - CYP19 reduction (MCF-7) }\end{array}$ & $\begin{array}{l}\text { (Licznerska et al. } \\
\text { 2015) }\end{array}$ \\
\hline $\begin{array}{l}\text { Prostate cancer cell lines ( } \mathrm{LNCaP}, \mathrm{C} 4-2 \\
\text { and PC-3) }\end{array}$ & $\begin{array}{l}1-20 \mu \mathrm{mol}(20 \text { for } \\
\text { myc, } 2.5 \text { and } 5 \\
\text { for pCSC) }\end{array}$ & $\begin{array}{l}\text { - Suppression in c myc } \\
\text { - pCSC inhibition } \\
\text { - ALDH1 reduction } \\
\text { - CD49f + reduction }\end{array}$ & $\begin{array}{l}\text { (Antosiewicz et al. } \\
\text { 2006) }\end{array}$ \\
\hline Breast cancer cell lines (MCF7 and SUM159) & $(1-5 \mu \mathrm{mol} / \mathrm{L})$ & $\begin{array}{l}\text { - Inhibition of proliferation } \\
\text { - Apoptosis induction } \\
\text { - ALDH reduction } \\
\text { - Size and number of primary mammospheres } \\
\quad \text { reduction }\end{array}$ & $\begin{array}{l}\text { (Kanematsu et al. } \\
\text { 2010) }\end{array}$ \\
\hline $\begin{array}{l}\text { Prostate cancer cell lines (DU145 and PC3) } \\
\text { with enriched CSC features, and primary } \\
\text { patient-derived prostate CSCs }\end{array}$ & $\begin{array}{l}\text { SFN and/or soluble } \\
\text { TRAIL }\end{array}$ & $\begin{array}{l}\text { - CSC properties reduction compared } \\
\text { to TRAIL treated } \\
\text { - TRAIL-induced NF-kB binding; CXCR4, } \\
\text { Jagged1, Notch } 1\end{array}$ & (Labsch et al. 2014) \\
\hline $\begin{array}{l}\text { Breast CSCs in vivo, a xenograft model of } \\
\text { SUM159 cells in NOD/SCID mice }\end{array}$ & $50 \mathrm{mg} / \mathrm{kg} / \mathrm{D}$ & $\begin{array}{l}\text { - ALDH-positive population reduction by }>50 \% \\
\text { - Tumorigenic potential reduction SFN, } 78 \text { to } 43 \% \text {, } \\
\text { TRAIL to } 38 \% \text {, and the double treatment to } 13 \%\end{array}$ & (Li et al. 2010) \\
\hline
\end{tabular}




\section{Perspective on combination therapy}

Cancer is a major global public health challenge. It has been suggested that $30-40 \%$ of cancer is preventable over time if appropriate food and nutrition is implemented in the diet (AICR 2007). One particular approach that has displayed effective results in oncology research is the use of a combination of therapeutics that synergistically target multiple pathways to formulate enhanced anti-cancer efficacy. The use of natural chemotherapeutic agents in combination therapy may serve as an alternate therapeutic intervention in cancer treatment and prevention. These agents partly deal with lessened financial and economic burden alluding to the fact that there is generally a vast supply of natural plant resources for extraction purposes. Many natural products have demonstrated potent anti-cancer properties and represent extensive chemical diversity due to the vast number of species and plants present on earth (Abhishek Bhanot and Noolvi 2011).

There has been extensive research on combination therapy for cancer treatment; for instance, bevacizumab in combination with an absorbable curcumin resulted in a reduction in tumor growth and inhibition of angiogenesis in HT29 colonic cancer cells (Yue et al. 2016). In another study, the combination of HDACi, MS-275, and demethylating agent, azacytidine, significantly induced apoptosis and inhibited tumor growth in mice with lung adenocarcinomas (Belinsky et al. 2011).

SFN has demonstrated chemopreventive properties with selective cytotoxicity in several types of cancer (Fig. 2) (Myzak and Dashwood 2006) that suggest SFN should be considered as a very effective chemopreventive agent for cancer. Moreover, combination therapies that combine SFN with other therapeutic agents favor a potential treatment modality for cancer (Kallifatidis et al. 2011, Islam et al. 2016). As an example, Kallifatidis et al. reported SFN to potentiate the anticancer effects of cisplatin, gemcitabine, doxorubicin or 5flurouracil on prostate cancer cell line MIA-PaCa2 while also increasing cytotoxicity of cancer stem cells (Kallifatidis et al. 2011). In some studies, it was shown that SFN can selectively target cancer cells and sensitize them to the cytotoxic effects of other anti-cancer drugs. For instance, administration of SFN enhanced the pro-apoptotic effects of oxaliplatin, a chemotherapeutic, on colorectal cancer cells because the combined agents simultaneously targeted both the extrinsic and intrinsic apoptotic pathways. This co-administration forced the cancer cells to undergo necrosis in addition to reducing apoptosis resistance (Kaminski et al. 2011).

Work from our lab illustrates the usefulness of SFN in combination with agents other than chemotherapeutics. Mokhtari et al. reported on the inhibitory effect of the combination of acetazolamide, a carbonic anhydrase inhibitor, and SFN on the growth and viability of bronchial carcinoid cell lines; $\mathrm{H} 727$ and H720 cell lines were used for in vitro and in vivo studies. SFN and AZ combination induced enhanced growth inhibition in lower dose concentration compared to either single agent. The combination of $\mathrm{AZ}$ and SFN effectively reduced clonogenic ability, 5-HT content, and the invasiveness of tumor cells (Mokhtari et al. 2013). Similarly, Islam et al. showed that the combination of acetazolamide and SFN inhibited tumor growth and induced apoptosis in a dose-dependent manner by blocking the PI3K/Akt pathway in bladder cancer cell lines HTB-9 and RT112 (H) (Islam et al. 2016). The combination of AZ + SFN displayed an additive role in the down-regulation of cell proliferation marker Ki-67 (Islam et al. 2016). Additionally, AZ potentiated SFN-induced apoptosis with higher efficacy compared to AZ or SFN alone (Mokhtari et al. 2013). Combinations of SFN with other carbonic anhydrase inhibitors or HDACi, such as benzenesulfonamide and synthetic MS-275, may display promising additive effects and therefore should be actively pursued. It should be noted that MS-275 also downregulates NFkB, thus potentially enhancing the anti-inflammatory response (Srivastava et al. 2010).

\section{Therapeutic value of sulforaphane}

The actual value of SFN in cancer chemoprevention and as a cancer therapeutic is still not fully elucidated. Theoretically, dietary isothiocyanates with their chemopreventive properties may prove valuable in the fight against cancer at all stages in tumor development. However, careful consideration should be taken when advising for the use of SFN with other chemotherapeutics because SFN has shown to reduce efficacy in certain cases. For instance, SFN mechanistically upregulates $\mathrm{Nrf} 2$ signaling. This mechanism may potentially reduce the effectiveness of other chemotherapeutics because the upregulation of Nrf2 results in the activation of phase 2 detoxifying enzymes, resulting in anti-apoptotic effects (Turrini et al. 2014).

SFN has shown promising results in numerous preclinical studies, however, more clinical studies are required to explore the efficacy as a chemopreventive or anti-cancer therapeutic agent in humans. In previous studies, SFN has shown to lack genotoxicity and be highly tolerable and safe in humans (Shapiro et al. 2006). It should be noted that different epidemiological or pre-clinical studies have used various sources of SFN, route, dose, duration and frequency. Therefore, a generally acceptable guideline for SFN administration is missing. Dietary doses of SFN have shown to modulate to some degree, xenobiotic-metabolizing enzyme systems and consequently, chemopreventive activity (Yoxall et al. 2005). However, a SFN concentration of $4.4 \mathrm{mg} / \mathrm{kg}$ per day was shown to have inhibitory growth effects on human cancer xenografts; corresponding to $308 \mathrm{mg}$ of SFN administered daily to a $70 \mathrm{~kg}$ individual (Herr and Buchler 2010). Moreover, Cornblatt et al. reported that a single dose of orally 
administered broccoli sprout ( $200 \mu \mathrm{mol}$ SFN) was well tolerated and was shown to exert chemoprotective efficacy in breast epithelial cells (Cornblatt et al. 2007). However, Atwell et al. found that twice-daily administration of SFN results in higher plasma concentrations compared to single dose administration, further supporting the need for a generally accepted guideline for SFN administration (Atwell et al. 2015).

Regarding the route and formulation of SFN administration, oral administration of fresh broccoli sprouts has shown to increase the absorption and consequently, the bioavailability of SFN in various studies (Atwell et al. 2015). An important factor to consider that affects the recovery of SFN from various preparations of cruciferous vegetables and dietary supplements is the difference in myrosinase activity (Clarke et al. 2011a, b). For instance, Shapiro et al. reported that the chewing of fresh broccoli sprouts increases the interaction of glucosinolates with myrosinase and consequently, increases the bioavailability of SFN in the body (Shapiro et al. 2001).

4NQO, 4-nitroquinoline-1-oxide; ALDH1, aldehyde dehydrogenase 1; ARE, antioxidant response elements; CSC, cancer stem cell; DNMT, methyltransferases; ECM, extracellular matrix; ESP, epithiospecifier protein; GSH, glutathione; GSTs, glutathione S-transferases; HDAC, histone deacetylase; HIF- $1 \alpha$, hypoxia-inducible factor- $1 \alpha$; MMPs, Matrix metalloproteinases; PEITC, Phenethyl isothiocyanate; RCC, renal cell carcinoma; SFN, sulforaphane; TIC, tumor initiating cell; TPA, 12-Otetradecanoylphorbol-13-acetate; TRAIL, Tumour Necrosis Factor-Related Apoptosis-Induced Ligand; VEGF, vascular endothelial growth factor; WCRF, World Cancer Research Fund.

Acknowledgments We thank Dr. Bikul Das, M.D., Ph.D. (The Forsyth Institute; Cambridge, MA) for critical review of the manuscript.

Author contributions Analysis and interpretation of the data: RBM, $\mathrm{NB}, \mathrm{HY}$; writing of the manuscript: RBM, NB, TSH, ZM, SK, PK and critical revision of the manuscript for intellectual content: RBM, NB, HY.

\section{Compliance with ethical standards}

Conflicts of interest None of the authors have any competing interests in the manuscript.

\section{References}

Abhishek Bhanot RS, Noolvi MN (2011) Natural sources as potential anti-cancer agents: a review. Intern J Phytomed 3:1

Aggarwal BB, Gehlot P (2009) Inflammation and cancer: how friendly is the relationship for cancer patients? Curr Opin Pharmacol 9(4):351369

AICR (2007) Food, nutrition, physical activity, and the prevention of cancer: a global perspective. World Cancer Research Fund/ American Institute for Cancer Research, Washington, DC

Alumkal JJ, Slottke R, Schwartzman J, Cherala G, Munar M, Graff JN, Beer TM, Ryan CW, Koop DR, Gibbs A, Gao L, Flamiatos JF,
Tucker E, Kleinschmidt R, Mori M (2015) A phase II study of sulforaphane-rich broccoli sprout extracts in men with recurrent prostate cancer. Investig New Drugs 33(2):480-489

Antosiewicz HA, Johnson DE, Singh SV (2006) Sulforaphane causes autophagy to inhibit release of cytochrome $\mathrm{C}$ and apoptosis in human prostate cancer cells. Cancer Res 66(11):5828-5835

Atwell LL, Hsu A, Wong CP, Stevens JF, Bella D, Yu TW, Pereira CB, Löhr CV, Christensen JM, Dashwood RH, Williams DE, Shannon J, Ho E (2015) Absorption and chemopreventive targets of sulforaphane in humans following consumption of broccoli sprouts or a myrosinase-treated broccoli sprout extract. Mol Nutr Food Res 59(3):242-233

Belinsky SA, Grimes MJ, Picchi MA, Mitchell HD, Stidley CA, Tesfaigzi Y, Channell MM, Liu Y, Casero RA Jr, Baylin SB, Reed MD, Tellez CS, March TH (2011) Combination therapy with vidaza and entinostat suppresses tumor growth and reprograms the epigenome in an orthotopic lung cancer model. Cancer Res 71(2):454-462

Benazzi C, Al-Dissi A, Chau CH, Figg WD, Sarli G, de Oliveira JT, Gärtner F (2014) Angiogenesis in spontaneous tumors and implications for comparative tumor biology. Sci World J 2014:1-17

Ben-Neriah Y, Karin M (2011) Inflammation meets cancer, with NFkappaB as the matchmaker. Nat Immunol 12(8):715-723

Bertl E, Bartsch H, Gerhauser C (2006) Inhibition of angiogenesis and endothelial cell functions are novel sulforaphane-mediated mechanisms in chemoprevention. Mol Cancer Ther 5(3):575-585

Choi S, Lew KL, Xiao H, Herman-Antosiewicz A, Xiao D, Brown CK, Singh SV (2007) D,L-Sulforaphane-induced cell death in human prostate cancer cells is regulated by inhibitor of apoptosis family proteins and Apaf-1. Carcinogenesis 28(1):151-162

Clarke JD, Hsu A, Riedl K, Bella D, Schwartz SJ, Stevens JF, Ho E (2011a) Bioavailability and inter-conversion of sulforaphane and erucin in human subjects consuming broccoli sprouts or broccoli supplement in a cross-over study design. Pharmacol Res 64(5): 456-463

Clarke JD, Hsu A, Yu Z, Dashwood RH, Ho E (2011b) Differential effects of sulforaphane on histone deacetylases, cell cycle arrest and apoptosis in normal prostate cells versus hyperplastic and cancerous prostate cells. Mol Nutr Food Res 55(7):999-1009

de Conti A, Kuroiwa-Trzmielina J, Horst MA, Bassoli BK, Chagas CE, Purgatto E, Cavalher FP, Camargo AA, Jordão AA Jr, Vannucchi H, Scolastici C, Ong TP, Moreno FS (2012) Chemopreventive effects of the dietary histone deacetylase inhibitor tributyrin alone or in combination with vitamin a during the promotion phase of rat hepatocarcinogenesis. J Nutr Biochem 8:860-866

Cornblatt BS, Ye L, Dinkova-Kostova AT, Erb M, Fahey JW, Singh NK, Chen MS, Stierer T, Garrett-Mayer E, Argani P, Davidson NE, Talalay P, Kensler TW, Visvanathan K (2007) Preclinical and clinical evaluation of sulforaphane for chemoprevention in the breast. Carcinogenesis 28(7):1485-1490

Cramer JM, Jeffery EH (2011) Sulforaphane absorption and excretion following ingestion of a semi-purified broccoli powder rich in glucoraphanin and broccoli sprouts in healthy men. Nutr Cancer 63(2): 196-201

Dinkova-Kostova AT, Jenkins SN, Fahey JW, Ye L, Wehage SL, Liby KT, Stephenson KK, Wade KL, Talalay P (2006) Protection against UV-light-induced skin carcinogenesis in SKH-1 high-risk mice by sulforaphane-containing broccoli sprout extracts. Cancer Lett 240(2):243-252

Disis ML (2010) Immune regulation of cancer. J Clin Oncol 28(29): 4531-4538

Egner PA, Chen JG, Zarth AT, Ng DK, Wang JB, Kensler KH, Jacobson LP, Muñoz A, Johnson JL, Groopman JD, Fahey JW, Talalay P, Zhu $\mathrm{J}$ et al (2014) Rapid and sustainable detoxication of airborne pollutants by broccoli sprout beverage: results of a randomized clinical trial in China. Cancer Prev Res 7(8):813-823 
Fahey JW, Talalay P, Kensler TW (2012) Notes from the field: "green" chemoprevention as frugal medicine. Cancer Prev Res 5(2):179188

Fraga MF, Ballestar E, Villar-Garea A, Boix-Chornet M, Espada J, Schotta G, Bonaldi T, Haydon C, Ropero S, Petrie K, Iyer NG, Pérez-Rosado A, Calvo E et al (2005) Loss of acetylation at Lys16 and trimethylation at Lys20 of histone $\mathrm{H} 4$ is a common hallmark of human cancer. Nat Genet 37(4):391-400

Gal-Yam EN, Saito Y, Egger G, Jones PA (2008) Cancer epigenetics: modifications, screening, and therapy. Annu Rev Med 59:267-280

Heiss E, Herhaus C, Klimo K, Bartsch H, Gerhauser C (2001) Nuclear factor kappa $\mathrm{B}$ is a molecular target for sulforaphane-mediated antiinflammatory mechanisms. J Biol Chem 276(34):32008-32015

Herr I, Buchler MW (2010) Dietary constituents of broccoli and other cruciferous vegetables: implications for prevention and therapy of cancer. Cancer Treat Rev 36(5):377-383

Hu R, Khor TO, Shen G, Jeong WS, Hebbar V, Chen C, Xu C, Reddy B, Chada K, Kong AN (2006) Cancer chemoprevention of intestinal polyposis in ApcMin/+ mice by sulforaphane, a natural product derived from cruciferous vegetable. Carcinogenesis 27(10):2038 2046

Huang TY, Chang WC, Wang MY, Yang YR, Hsu YC (2012) Effect of sulforaphane on growth inhibition in human brain malignant glioma GBM 8401 cells by means of mitochondrial-and MEK/ERKmediated apoptosis pathway. Cell Biochem Biophys 63(3):247-259

Islam SS, Mokhtari RB, Akbari P, Hatina J, Yeger H, Farhat WA (2016) Simultaneous targeting of bladder tumor growth, survival, and epithelial-to-mesenchymal transition with a novel therapeutic combination of acetazolamide (AZ) and Sulforaphane (SFN). Target Oncol 11(2):209-227

Jaenisch R, Bird A (2003) Epigenetic regulation of gene expression: how the genome integrates intrinsic and environmental signals. Nat Genet:245-254

Jee HG, Lee KE, Kim JB, Shin HK, Youn YK (2011) Sulforaphane inhibits oral carcinoma cell migration and invasion in vitro. Phytother Res 25(11):1623-1628

Jeffery EH, Keck AS (2008) Translating knowledge generated by epidemiological and in vitro studies into dietary cancer prevention. Mol Nutr Food Res 52(Suppl 1):S7-17

Jost PJ, Ruland J (2007) Aberrant NF-kappaB signaling in lymphoma: mechanisms, consequences, and therapeutic implications. Blood 109(7):2700-2707

Kallifatidis G, Labsch S, Rausch V, Mattern J, Gladkich J, Moldenhauer G, Büchler MW, Salnikov AV, Herr I (2011) Sulforaphane increases drug-mediated cytotoxicity toward cancer stem-like cells of pancreas and prostate. Mol Ther 19(1):188-195

Kaminski BM, Weigert A, Brune B, Schumacher M, Wenzel U, Steinhilber D, Stein J, Ulrich S (2011) Sulforaphane potentiates oxaliplatin-induced cell growth inhibition in colorectal cancer cells via induction of different modes of cell death. Cancer Chemother Pharmacol 67(5):1167-1178

Kanematsu S, Uehara N, Miki H, Yoshizawa K, Kawanaka A, Yuri T, Tsubura A (2010) Autophagy inhibition enhances sulforaphaneinduced apoptosis in human breast cancer cells. Anticancer Res 30(9):3381-3390

Kassahun K, Davis M, Hu P, Martin B, Baillie T (1997) Biotransformation of the naturally occurring isothiocyanate sulforaphane in the rat: identification of phase I metabolites and glutathione conjugates. Chem Res Toxicol 10(11):1228-1233

Kim DH, Sung B, Kang YJ, Hwang SY, Kim MJ, Yoon JH, Im E, Kim ND (2015) Sulforaphane inhibits hypoxia-induced HIF-1alpha and VEGF expression and migration of human colon cancer cells. Int $\mathrm{J}$ Oncol 47(6):2226-2232

Kolonel LN, Hankin JH, Whittemore AS, Wu AH, Gallagher RP, Wilkens LR, John EM, Howe GR, Dreon DM, West DW, Paffenbarger RS Jr (2000) Vegetables, fruits, legumes and prostate cancer: a multiethnic case-control study. Cancer Epidemiol Biomark Prev 9(8):795-804

Kumar A, Sabbioni G (2010) New biomarkers for monitoring the levels of isothiocyanates in humans. Chem Res Toxicol 23(4):756-765

Kwak MK, Wakabayashi N, Kensler TW (2004) Chemoprevention through the Keap1-Nrf2 signaling pathway by phase 2 enzyme inducers. Mutat Res 555(1-2):133-148

Labsch S, Liu L, Bauer N, Zhang Y, Aleksandrowicz E, Gladkich J et al (2014) Sulforaphane and TRAIL induce a synergistic elimination of advanced prostate cancer stem-like cells. Int J Oncol 44(5):1470 1480

Langouet S, Furge LL, Kerriguy N, Nakamura K, Guillouzo A, Guengerich FP (2000) Inhibition of human cytochrome P450 enzymes by 1,2-dithiole-3-thione, oltipraz and its derivatives, and sulforaphane. Chem Res Toxicol 13(4):245-252

Lee CS, Cho HJ, Jeong YJ, Shin JM, Park KK, Park YY, Bae YS, Chung IK, Kim M, Kim CH, Jin F, Chang HW, Chang YC (2015) Isothiocyanates inhibit the invasion and migration of $\mathrm{C} 6$ glioma cells by blocking FAK/JNK-mediated MMP-9 expression. Oncol Rep 34(6):2901-2908

Li Y, Zhang T, Korkaya H, Liu S, Lee HF, Newman B, Yu Y, Clouthier SG, Schwartz SJ, Wicha MS, Sun D (2010) Sulforaphane, a dietary component of broccoli/broccoli sprouts, inhibits breast cancer stem cells. Clin Cancer Res 16(9):2580-2590

Licznerska B, Szaefer H, Matuszak I, Murias M, Baer-Dubowska W (2015) Modulating potential of L-sulforaphane in the expression of cytochrome p450 to identify potential targets for breast cancer chemoprevention and therapy using breast cell lines. Phytother Res 29(1):93-99

Luang-In V, Narbad A, Nueno-Palop C, Mithen R, Bennett M, Rossiter JT (2014) The metabolism of methylsulfinylalkyl- and methylthioalkyl-glucosinolates by a selection of human gut bacteria. Mol Nutr Food Res 58(4):875-883

Matusheski NV, Jeffery EH (2001) Comparison of the bioactivity of two glucoraphanin hydrolysis products found in broccoli, sulforaphane and sulforaphane nitrile. J Agric Food Chem 49(12):5743-5749

Mokhtari RB, Kumar S, Islam SS, Yazdanpanah M, Adeli K, Cutz E, Yeger H (2013) Combination of carbonic anhydrase inhibitor, acetazolamide, and sulforaphane, reduces the viability and growth of bronchial carcinoid cell lines. BMC Cancer 13:378

Myzak MC, Dashwood RH (2006) Chemoprotection by sulforaphane: keep one eye beyond Keap1. Cancer Lett 233(2):208-218

Nian H, Delage B, Ho E, Dashwood RH (2009) Modulation of histone deacetylase activity by dietary isothiocyanates and allyl sulfides: studies with sulforaphane and garlic organosulfur compounds. Environ Mol Mutagen 50(3):213-221

Reece JC, Chan YF, Herbert J, Gralow J, Fann JR (2013) Course of depression, mental health service utilization and treatment preferences in women receiving chemotherapy for breast cancer. Gen Hosp Psychiatry 35(4):376-381

Rudolf K, Cervinka M, Rudolf E (2014) Sulforaphane-induced apoptosis involves p53 and p38 in melanoma cells. Apoptosis 19(4):734-747

Shapiro TA, Fahey JW, Wade KL, Stephenson KK, Talalay P (2001) Chemoprotective glucosinolates and isothiocyanates of broccoli sprouts: metabolism and excretion in humans. Cancer Epidemiol Biomark Prev 10(5):501-508

Shapiro TA, Fahey JW, Dinkova-Kostova AT, Holtzclaw WD, Stephenson KK, Wade KL, Ye L, Ye L, Talalay P (2006) Safety, tolerance, and metabolism of broccoli sprout glucosinolates and isothiocyanates: a clinical phase I study. Nutr Cancer 55(1):53-62

Sheth SH, Johnson DE, Kensler TW, Bauman JE (2015) Chemoprevention targets for tobacco- related head and neck cancer: past lessons and future directions. Oral Oncol 51(6):557-564

Shibata A, Nakagawa K, Yamanoi H, Tsuduki T, Sookwong P, Higuchi O, Kimura F, Miyazawa T (2010) Sulforaphane suppresses 
ultraviolet B-induced inflammation in HaCaT keratinocytes and HR-1 hairless mice. J Nutr Biochem 21(8):702-709

Singh AV, Xiao D, Lew KL, Dhir R, Singh SV (2004) Sulforaphane induces caspase-mediated apoptosis in cultured PC-3 human prostate cancer cells and retards growth of PC-3 xenografts in vivo. Carcinogenesis 25(1):83-90

Singh A, Sharma N, Ghosh M, Park Y, Jeong D (2017) Emerging importance of dietary phytochemicals in fight against cancer: role in targeting cancer stem cells. Crit Rev Food Sci Nutr 57(16):34493463

Srivastava VK, Hill DC (1974) Glucosinolate hydrolytic products given by Sinapis alba, and Brassica napus thioglucosidases. Phytochemistry 13: 1043-1046

Srivastava RK, Kurzrock R, Shankar S (2010) MS-275 sensitizes TRAIL-resistant breast cancer cells, inhibits angiogenesis and metastasis, and reverses epithelial-mesenchymal transition in vivo. Mol Cancer Ther 9(12):3254-3266

Thejass P, Kuttan G (2006) Antimetastatic activity of Sulforaphane. Life Sci 78(26):3043-3050

Tortorella SM, Royce SG, Licciardi PB15V, Karagiannis TC (2015) Dietary Sulforaphane in cancer chemoprevention: the role of epigenetic regulation and HDAC inhibition. Antioxid Redox Signal 22(16): $1382-1424$

Tsuchiya Y, Endo Y, Sato H, Okada Y, Mai M, Sasaki T, Seiki M (1994) Expression of type-IV collagenases in human tumor cell lines that can form liver colonies in chick embryos. Int J Cancer 56(1):46-51

Turrini E, Ferruzzi L, Fimognari C (2014) Natural compounds to overcome cancer chemoresistance: toxicological and clinical issues. Expert Opin Drug Metab Toxicol 10(12):1677-1690

Vyas AR, Moura MB, Hahm ER, Singh KB, Singh SV (2016) Sulforaphane inhibits c-Myc-mediated prostate cancer stem-like traits. J Cell Biochem 117(11):2482-2495

Wu QJ, Yang Y, Vogtmann E, Wang J, Han LH, Li HL, Xiang YB (2013) Cruciferous vegetables intake and the risk of colorectal cancer: a meta-analysis of observational studies. Ann Oncol 24(4):1079-1087

Wu S, Gao Q, Zhao P, Gao Y, Xi Y, Wang X, Liang Y, Shi H, Ma Y (2016) Sulforaphane produces antidepressant- and anxiolytic-like effects in adult mice. Behav Brain Res 301:55-62
Xu T, Ren D, Sun X, Yang G (2012) Dual roles of sulforaphane in cancer treatment. Anti Cancer Agents Med Chem 12(9):1132-1142

Youn HS, Kim YS, Park ZY, Kim SY, Choi NY, Joung SM, Seo JA, Lim KM, Kwak MK, Hwang DH, Lee JY (2010) Sulforaphane suppresses oligomerization of TLR4 in a thiol- dependent manner. J Immunol 184(1):411-419

Yoxall V, Kentish P, Coldham N, Kuhnert N, Sauer MJ, Ioannides C (2005) Modulation of hepatic cytochromes P450 and phase II enzymes by dietary doses of sulforaphane in rats: implications for its chemopreventive activity. Int J Cancer 117(3):356-362

Yuan JM, Gago-Dominguez M, Castelao JE, Hankin JH, Ross RK, Yu MC (1998) Cruciferous vegetables in relation to renal cell carcinoma. Int J Cancer 77(2):211-216

Yue GG-L, Kwok H-F, Lee JK-M, Jiang L, Wong EC-W, Gao S, Wong H-L, Li L, Chan K-M, Leung P-C, Fung K-P, Zuo Z, Lau CB-S (2016) Combined therapy using bevacizumab and turmeric ethanolic extract (with absorbable curcumin) exhibited beneficial efficacy in colon cancer mice. Pharmacol Res 111:43-57

Zhang Y, Talalay P, Cho CG, Posner GH (1992) A major inducer of anticarcinogenic protective enzymes from broccoli: isolation and elucidation of structure. Proc Natl Acad Sci U S A 89(6):2399-2403

Zhang SM, Hunter DJ, Rosner BA, Giovannucci EL, Colditz GA, Speizer FE, Willett WC (2009) Intakes of fruits, vegetables, and related nutrients and the risk of non-Hodgkin's lymphoma among women. Cancer Epidemiol Biomark Prev 9(5):477-485

Zhang Z, Atwell LL, Farris PE, Ho E, Shannon J (2016a) Associations between cruciferous vegetable intake and selected biomarkers among women scheduled for breast biopsies. Public Health Nutr 7:1288-1295

Zhang Z, Li C, Shang L, Zhang Y, Zou R, Zhan Y, Zou R, Zhan Y, Bi B (2016b) Sulforaphane induces apoptosis and inhibits invasion in U251MG glioblastoma cells. Spring 5:235

Zuryn A, Litwiniec A, Safiejko-Mroczka B, Klimaszewska-Wisniewska A, Gagat M, Krajewski A, Gackowska L, Grzanka D (2016) The effect of sulforaphane on the cell cycle, apoptosis and expression of cyclin D1 and p21 in the A549 non-small cell lung cancer cell line. Int J Oncol 48(6):2521-2533 